\title{
Repeat Microvascular Decompression for Trigeminal Neuralgia After Initial Microvascular Decompression: Three Cases and Literature Review
}

ISSN: 2637-7748

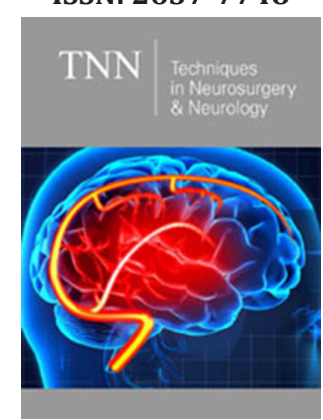

*Corresponding author: Ellen Frydenberg, Suite 1405, St Vincents Private Hospital, 406 Victoria Street, Darlinghurst, NSW 2010, Australia

Submission: 㘹 October 21, 2019

Published: 佂 November 13, 2019

Volume 3 - Issue 1

How to cite this article: Frydenberg E, Xia M, Tomlinson S, Steel T. Repeat Microvascular Decompression for Trigeminal Neuralgia After Initial Microvascular Decompression: Three Cases and Literature Review. Tech Neurosurg Neurol.3(1).TNN.000553.2019. DOI: 10.31031 /TNN.2019.03.000553

Copyright@ Ellen Frydenberg, This article is distributed under the terms of the Creative Commons Attribution 4.0 International License, which permits unrestricted use and redistribution provided that the original author and source are credited.

\author{
Frydenberg $\mathrm{EM}^{1,2,3 *}$, Xia $\mathbf{M}^{1,2}$, Tomlinson $\mathrm{SE}^{1,2,4}$ and Steel $\mathrm{TR}^{1,2,3}$ \\ ${ }^{1}$ St Vincent's General Hospital, Darlinghurst, Australia \\ ${ }^{2}$ St Vincent's Private Hospital, Darlinghurst, Australia \\ ${ }^{3}$ University of NSW, Australia \\ ${ }^{4}$ University of Sydney, Australia
}

\begin{abstract}
Trigeminal neuralgia (TN) is a debilitating disorder manifesting as intense lancinating pain in in the distribution of the trigeminal nerve. Microvascular decompression (MVD) of the trigeminal nerve has become the first choice for patients with TN refractory to medication as it is effective and durable with initial pain relief reported as high as $98 \%$ however recurrence of TN pain has been reported as high as $47 \%$ at 8.5 years follow up. The long-term efficacy remains significantly higher than percutaneous neuroablative techniques with recurrence rates reported as high as $75 \%$ within 5 years. Recurrence of TN following MVD poses a management challenge with no evidence-based guidelines for treatment. Initial series of repeat MVD describe high complication rates which, combined with high negative exploration rates for neurovascular conflicts, led authors to favor percutaneous interventions for medically refractory cases. This report describes three cases and reviews the current literature. Recent literature indicates that the predominant causes of recurrent TN are adhesions around the graft, the development of a Teflon granuloma or recurrent vascular compression. The cases reported here support the recommendation that repeat MVD can be highly effective in alleviating recurrent TN whether the recurrent compression is due to the prosthesis or recurrent vascular compression.
\end{abstract}

Keywords: Trigeminal neuralgia; Microvascular decompression; Facial pain

\section{Introduction}

Trigeminal neuralgia (TN) is a debilitating disorder manifesting as intense lancinating pain in in the distribution of the trigeminal nerve [1]. Vascular compression of the root entry zone of (REZ) of the nerve is thought to account for the majority of cases of TN [2]. Pharmacological treatment with medication such as carbamazepine is first line of therapy. Microvascular decompression (MVD) of the trigeminal nerve has become the first choice for patients with TN refractory to medication as it is an effective treatment option with initial pain relief reported as high as $98 \%$ [3-5].

Longer follow-up post MVD shows ongoing risks of recurrence several years post operatively, with Matsushima reporting a 17.1\% recurrence rate at 2 years, Barker reporting $30 \%$ at 6.2 years and Burchiel reporting $47 \%$ at 8.5 years [6-8]. The long-term efficacy of MVD however remains significantly higher than percutaneous neuro-ablative techniques with patient undergoing percutaneous rhizotomy having a recurrence rate of $75 \%$ within 5 years [9].

Recurrence of trigeminal neuralgia following MVD poses a management challenge with no evidence-based guidelines for treatment. Repeat MVD has been advocated for medically intractable cases [10]. Repeat MVD has a lower rate of finding vascular compression than at initial surgery [10]. Initial series of repeat MVD were associated with high complication rates prompting authors not to recommend repeat surgery $[10,11]$. Percutaneous procedures such as glycerol or radiofrequency rhizotomy were instead initially favored, however recent series have reported good outcomes with repeat MVD [6,12-16]. 
Recurrent vascular compression after absorption or slippage of an implant, scarring around an implant, and recanalization of a vein were reported in early series(10). In recent series the role of the interposition graft material has been implicated $(6,13-15)$. This report presents three illustrative cases of recurrent TN and reviews the current literature.

\section{Methods}

A retrospective case review of three patients in whom a second trigeminal nerve MVD had been performed for recurrence of trigeminal neuralgia after a previous MVD was undertaken.

\section{Clinical Details}

Initial surgery in all patients was following a diagnosis of classical TN due to a vascular loop in contact with the nerve at the REZ. Initial surgery had been undertaken by other surgeons in Patients 1 and 2. Patient 1 had interposition of Teflon and Patient 2 had insertion of a silastic sheet. Initial surgery in Patient 3 was performed by the senior author (TS) with Teflon graft. All patients experienced initial alleviation of pain. Recurrent TN reoccurred at 4, 10 and 9 years after initial surgery. All 3 underwent repeat MVD by the senior author (TS) with complete alleviation of pain.

\section{Patient 1}

A 68-year old female presented with right sided TN in 2009 and underwent MVD in 2012 with a Teflon (polytetrafluorethylene) pledget with complete alleviation of pain. Recurrent right sided TN occurred in 2017; 5 years after initial surgery. The recurrent pain was typical TN, in a V2/3 distribution. MRI scan showed a hypointense mass in contact with REZ of the right trigeminal nerve (Figure 1). Repeat MVD was performed in December 2017. At surgery the previously placed PTFE patch was found to be adherent, compressing the nerve (Figure 2a). There was no recurrent vascular compression. The Teflon was dissected from the nerve and removed (Figure 2b). As there was no vascular compression, no new material was placed. She had complete resolution of pain and remains off all analgesia 24 months after surgery.

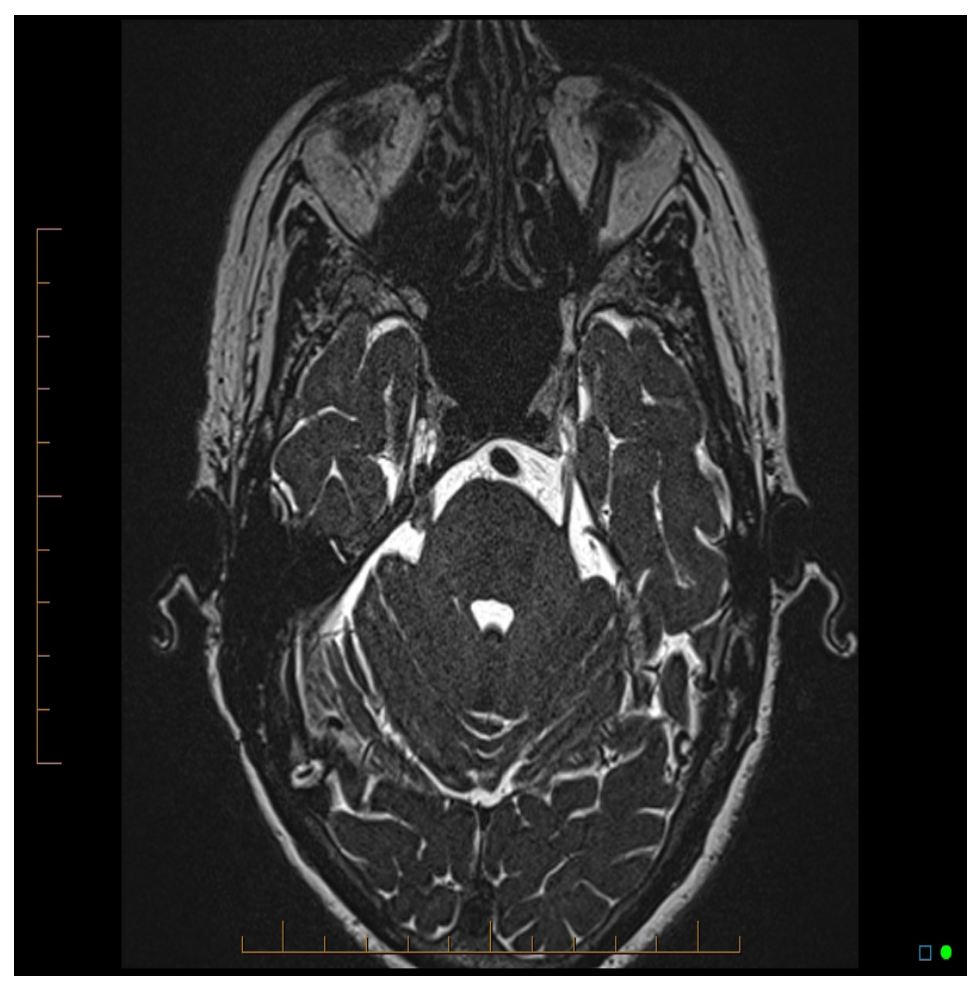

Figure 1: Case 1. 68-year-old female with recurrent right TN. 5 years after initial surgery. Material is seen around the right trigeminal nerve. No recurrent neurovascular compression is seen.

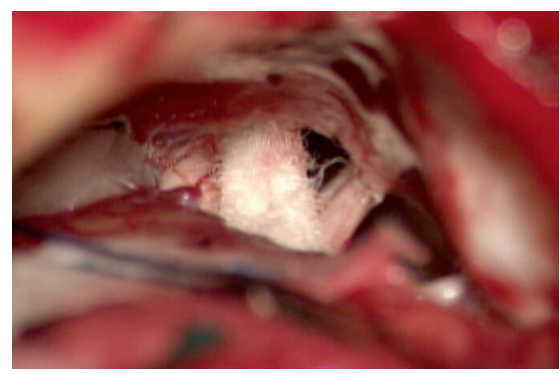

Figure 2a: Case 1. Nerve compression by Teflon pledgets from previous surgery. 


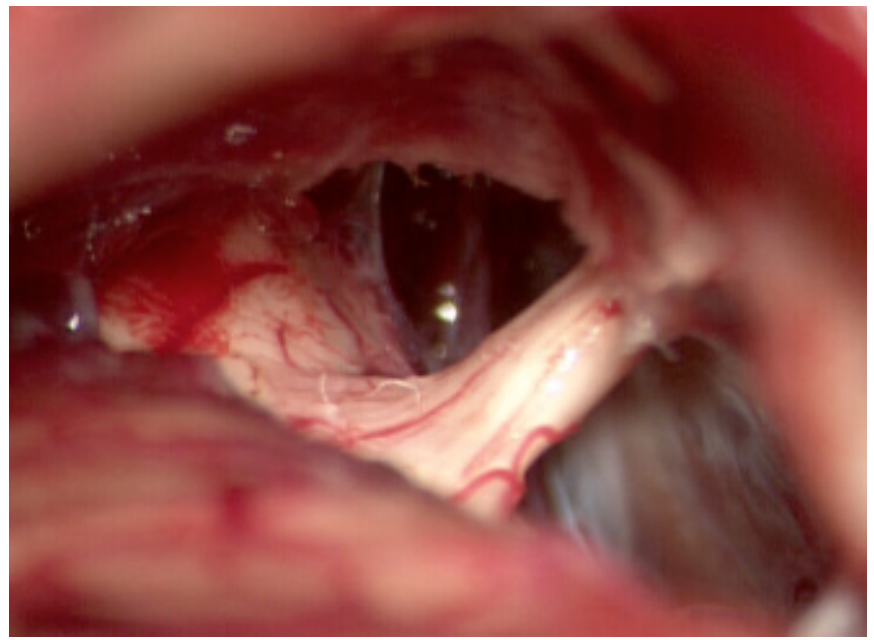

Figure 2b: Case 1. Trigeminal nerve free, indentation still visible

\section{Patient 2}

A 79-year-old female developed left TN in 2004 and underwent MVD in 2007. At surgery a silastic sheet was placed between the artery and the nerve. She had complete relief of pain until 2017, 10 years after initial surgery. Cerebral CT showed silastic sheeting in the left CP angle (Figure 3). Cerebral MRI showed 2 silastic sheets in the left cerebellopontine angle adjacent to the mid cisternal portion of the left trigeminal nerve. No compressive vascular loop was evident (Figure 4). Pain was progressive and unresponsive to medical therapy. Repeat MVD was performed. At surgery, the silastic sheet was found compressing and indenting the nerve (Figure 5a). It was removed (Figure 5b). There was no vascular compression. She had complete resolution of pain 2 years after repeat MVD.

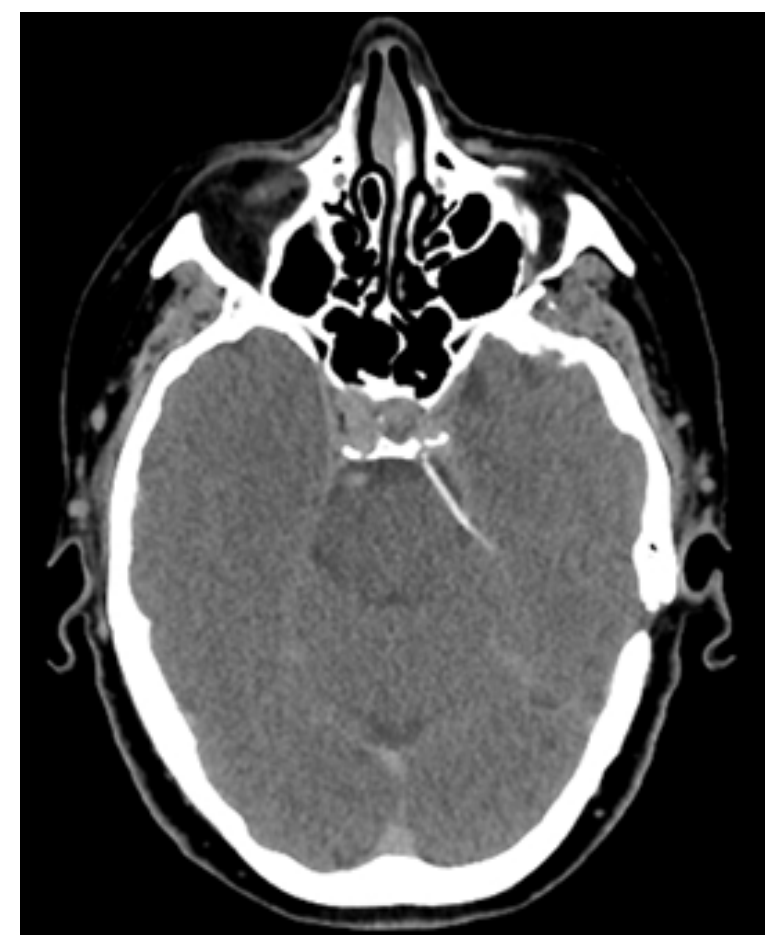

Figure 3: Case 2. Large silastic sheet in the left pontine region.

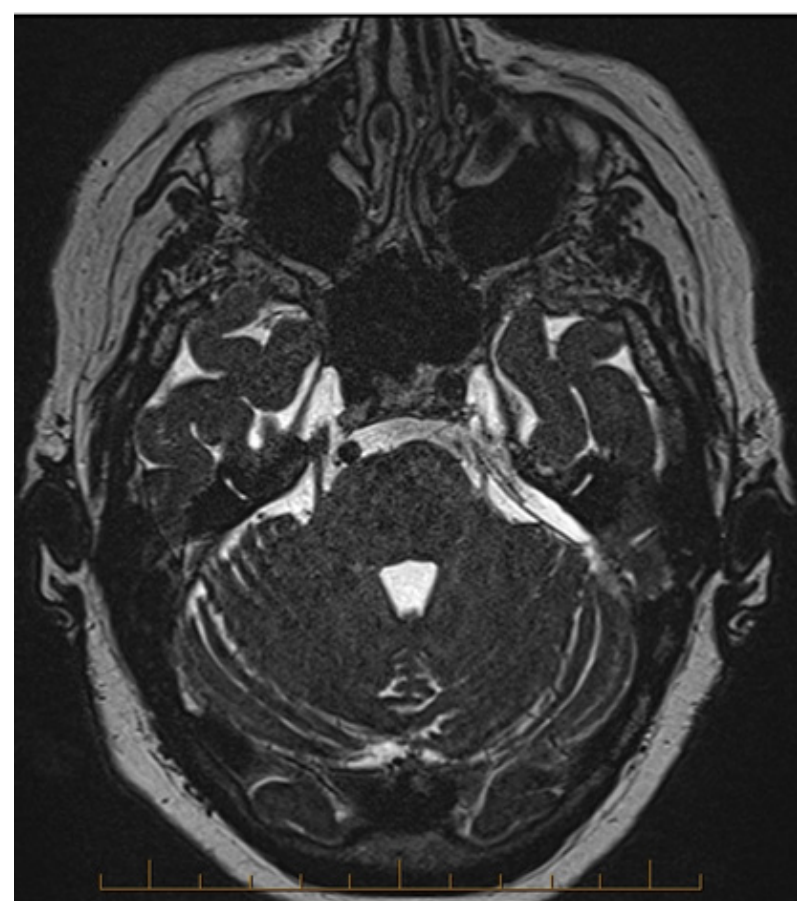

Figure 4: Case 2. No vascular loop evident, but silastic sheet lying adjacent to mid-cisternal portion of the left trigeminal nerve.

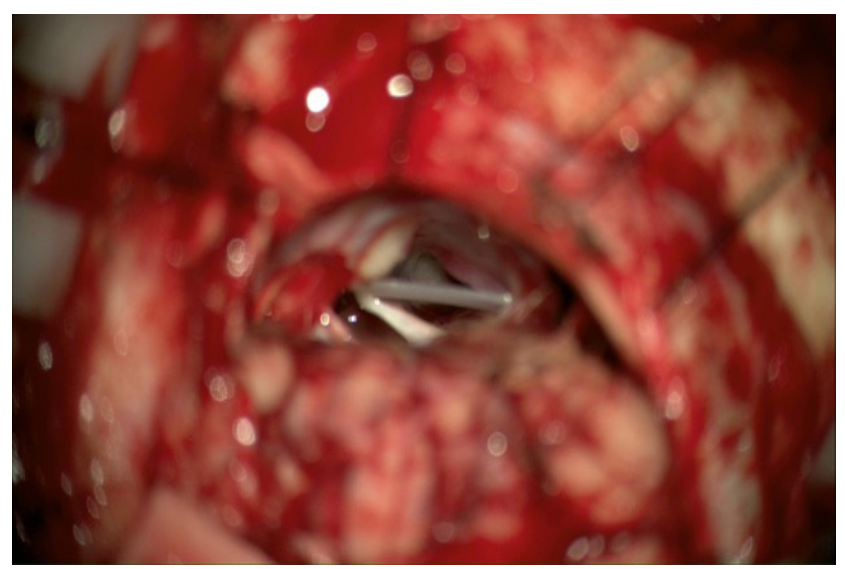

Figure 5a: Case 2. Silastic sheet indenting the trigeminal nerve

\section{Patient 3}

A 69-year-old male developed right V2 TN in 2006. MVD was performed in February 2009 by the senior author (TS) with complete resolution of pain. In 2012 recurrent TN developed after a right total knee replacement during which an intraspinal anesthetic was performed. Pain resolved after 2 weeks. A left knee replacement was performed in May 2015 with another intraspinal anesthetic. Following this he developed recurrent right V2 TN which failed to resolve. Cerebral MRI scan showed the Teflon pledget adjacent to the cisternal segment of the right trigeminal nerve (Fig 6). The right SCA lay in contact with the lateral margin of the material. Repeat MVD was performed. Intraoperatively, recurrent compression of the REZ of the trigeminal nerve was found due to a descending loop of the superior cerebellar artery. The pledget remained in good position with the new vessel loop lying posterior to the interposed 
material compressing the REZ. The pledget was divided using micro dissection and the SCA was mobilized superiorly. Two new PTFE pledgets were placed. It was hypothesized that the lumbar punctures performed at the time of the spinal anesthetics caused the development of CSF low pressure flow which caused the vessel loop to descend onto the nerve. This association has not been reported previously. Following the repeat surgery, the patient ceased all analgesia and has complete pain relief 4 years following repeat MVD.

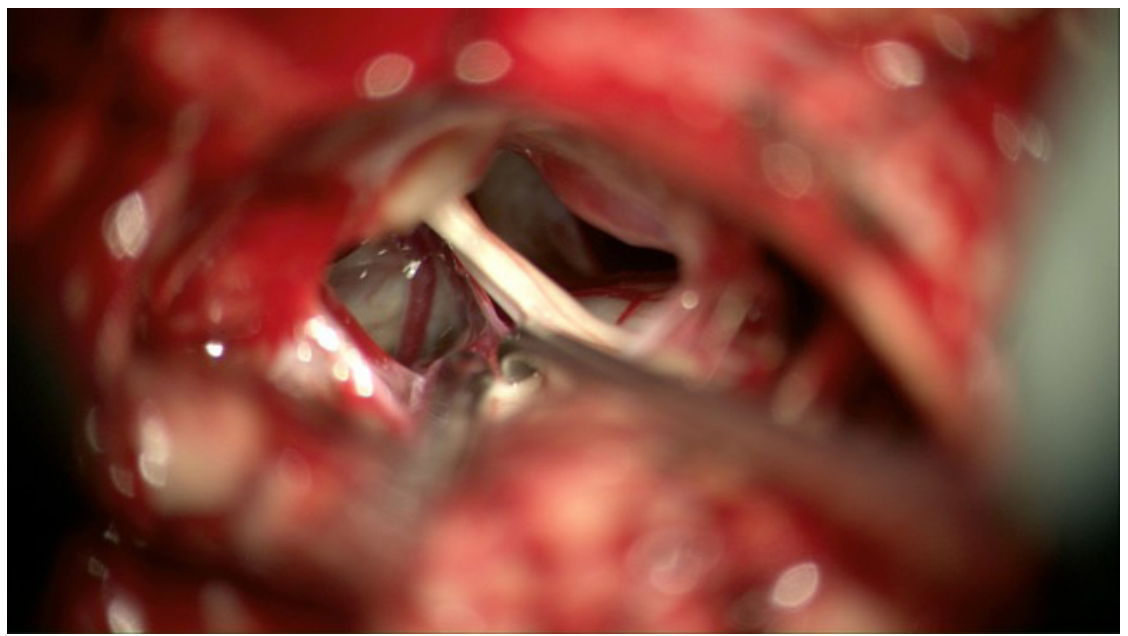

Figure 5b: Case 2. Silastic sheet removed, nerve free of compression. Nerve indentation visible from longstanding nerve compression.

\section{Discussion}

Trigeminal neuralgia is a devastating condition which severely interferes with quality of life and work efficiency. Patients with TN are often incapacitated by unpredictable lancinating pain in the distribution of the trigeminal nerve. Multiple modalities of treatment are recommended. Medical treatment is first line therapy and is often highly effective. Microvascular decompression (MVD) is considered first line surgical treatment for patients with medically intractable TN. Improved anatomical knowledge, microsurgical and anaesthetic techniques have now made MVD safer and more effective than in previous decades. It has higher rates of complete pain alleviation and lower rates of post-operative complications than other surgical treatments. Patients with TN are almost always immediately cured after the MVD procedure [4]. It is now the most commonly performed operation for TN in the USA and is recommended for MRI demonstrable neurovascular compression as first line therapy $[2,12,17,18]$.

Because MVD is more invasive than percutaneous procedures for TN, its safety in elderly patients has been questioned [4]. Early series reported an increase in complications in elderly patients, however, recent studies including a meta-analysis have reported no difference in complication rates between younger and older patients [4]. The operation is technically easier in the elderly as cerebellar atrophy leads to less need for retraction [19]. Complications from MVD are rare in experienced hands with only a $4 \%$ complication rate reported [4]. Post-operative trigeminal nerve deficits, facial nerve weakness, hearing loss, aseptic meningitis, hydrocephalus, CSF leak and cerebellar stroke are all recognized complications [4].

Although the immediate success rate of MVD is high, recurrent TN occurs at longer term follow-up $[7,8,10,20,21]$. Recurrence rates of TN after MVD range from 3-47\% depending on the duration of follow up [6]. In the largest review of 1204 patients with followup of more than 6 years, $30 \%$ of patients developed recurrent pain [7]. Burchill [8] reported $47 \%$ of patients developed recurrence at a mean of 8.5 years. Choo et al. [22] reported $14 \%$ recurrence at one-year follow-up. The variation in recurrence rate of $\mathrm{TN}$ may result from the variation in surgical technique between different surgeons, the duration of the follow-up period, and the definition of recurrence [13]. Many authors report the recurrence of TN symptoms after MVD usually developing within the first two years while later recurrence is rare with an annual recurrence of less than $1.1 \%$ [7]. In our patient cohort pain recurred at 4, 9 and 11 years after their initial procedure.

The likelihood of recurrence of TN is variable and several causes have been reported. Initial series reported most recurrence occurred within 2 years of surgery $[5,13,23,24]$ In initial series prior to the routine use of MRI scans and stereotactic navigation, inadequate decompression due to inability to visualize the cisternal portion of the trigeminal nerve and movement or atrophy of the graft were felt to be responsible.

In early series interposition graft was of various materials such as muscle, lyophilized dura, Gel foam or polyvinyl alcohol foam (Ivalon sponge) $[4,12]$. In recent series with Teflon, graft migration is considered rare [19]. In our 3 cases the graft remained in good position on preoperative MRI.

Different techniques applied for the transposition of the conflicting vessels led to variation in outcome. Jannetta et al reexplored 51 cases of recurrent TN after MVD and recurrent vascular compression was found in 42 cases (82\%) leading them to presume that vascular compression is the major cause of recurrence(11). 
Kureshi et al. [10] re-explored 23 cases of recurrent TN after the prior MVD and found recurrent vascular compression in $30 \%$ of cases. Choo et al. [22] repeated posterior fossa exploration and found vascular compression in 11 cases (35\%). There was no cause found for recurrent pain in 16 patients (52\%), Teflon adhesions were found in 4 (13\%) [22]. Most of the recurrent neural compressions were due to adhesions around the implant. Teflon granulomas have been implicated in recurrent TN $[10,11,22,25]$. Yang et al. [13] studied 15 patients with recurrent TN and found 10 cases had adhesions causing compression, 4 had recurrent vascular compression and slippage of the Teflon was found in one case. Yang concluded Teflon adhesions should be considered the most likely cause of recurrent symptoms after MVD [13].

The first report of Teflon as an interposition graft was in 1990 by Ammar et al. (26). Prior to the use of Teflon, muscle, Ivalon sponge, cotton and other tissues were used. Teflon was felt to be superior as an interposition graft as it was inert and non-absorbable. Teflon induced granulomas have subsequently been reported as causing recurrent TN [25]. Matsushima et al found that in all of their 63 explored cases, adhesion of the Teflon to the nerve was felt to be responsible [6].

Using any kind of implant in the interposition technique can cause recurrent compression as adhesions occur. In 1996, Fujimaki reported 2 cases of recurrent TN caused by direct compression from the inserted prosthesis, an association not previously described [14]. A Teflon and a polyethylene interposition implant caused recurrent pain 17 months and 9 years after initial surgery [14]. Both patients reported complete relief of pain following the surgery with removal of the implants [14]. At repeat surgery, the Teflon had become fibrotic and firm as in our case no 1 . The Polyethylene implant was causing direct compression as in our Patient 2.

To avoid recurrent compression some authors have recommend sling techniques which completely decompress the nerve and elevate the vessel to avoid any contact with the nerve itself.
Matsushima reported 6 patients treated with re-do surgery using the sling technique in 2000 [6]. In all 6 cases, the interposed Teflon from the initial surgery was confirmed to adhere to the trigeminal nerve, and in one case a granuloma had formed [6]. There were no cases of recurrent vascular compression [6]. All Matsushima's repeat sling MVDs resulted in a good outcome with alleviation of pain [6]. Minor complications were observed in 4 cases including facial sensory disturbance and reduced hearing in one case [6]. They concluded repeat MVD for recurrent TN is safe and effective and recommend a second MVD for patients if they have had an interval period free of pain, particularly if the compression in the original surgery was arterial as in our 3 cases [6]. They recommended the sling retraction technique be used for the initial MVD [6].

Other authors do not recommend sling techniques as the wider access required to manipulate the vessel and suspend it to the dura potentially endangers the vessels and requires more retraction of the cerebellum. This increases the risk of 7th and 8th cranial nerve deficits. The offending vessels are transposed and anchored using vascular tapes, thread, aneurysm clips and Tisseal glue. The vessel is fixed to the inferior surface of the tentorium or the adjacent petrous bone depending on the anatomy of the vessel.

Ugwuanyi and Kitchen reported repeat surgery in 6 patients in 2010 [12]. They found recurrent vascular compression in 5 cases and dense arachnoid adhesion around the Teflon in one [12]. They recommended there should be little hesitation undertaking re-do surgery in patients suffering recurrent TN when they have had a prolonged pain free period following the first surgery [12]. They felt the increased exposure needed for the sling techniques increased the potential complications of hearing loss and facial paresis and did not recommend its routine use [12]. In our 3 patients the graft material was seen in close approximation to the nerve on preoperative MRI. While no obvious vascular compression was seen it was impossible to assess vascular compression pre-operatively due the presence of interposition material [Figure 1,4 \& 6].

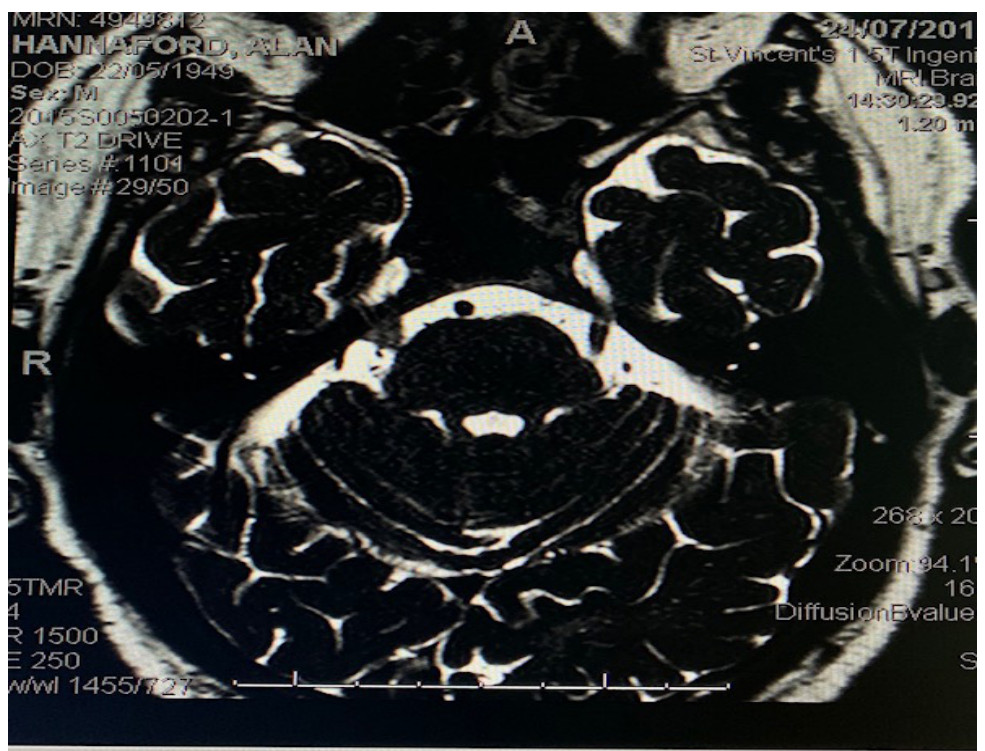

Figure 6: 69-year-old male with recurrent TN. MRI Fiesta views 6 years after initial surgery showing previously placed material seen around the right Trigeminal nerve. 
Recurrence of TN symptoms after a previous MVD remains a significant challenge. The development of adhesions between the foreign material and the neighboring vessels makes the second MVD more difficult and hazardous. Yang et al. [13] in their 15 cases showed good results and they did not see higher incidence of complication. One of their 15 patients developed hearing loss which was permanent. Their mean follow-up period was 3.2 years in which time no patient experienced recurrence [13].

Because of a higher number of complications and difficulty of the surgery some authors have in initial series recommended against repeat MVD. In 1998 Kureshi [10] reported on 23 repeat explorations for persistent or recurrent TN and found no evidence of implant migration. Recurrent compression was found in 7 patients, 5 from a scarred implant, one from an artery and one from a bony ridge [10]. Complete relief of pain was obtained in 15 cases (65\%), partial relief was achieved in six patients and two patients had no relief [10]. Because of a high number of complications (30\%) the authors recommended against repeat MVD, despite achieving significant pain relief in all but 2 patients [10]. They instead recommended percutaneous ablative procedures be performed [10]. They quoted Tew who reported a 98\% response in alleviating TN with percutaneous radio-frequency neurotomy when MVD fails [27]. Kureshi [10] recommended re-exploration only if it was felt the vascular compression had not been not recognized during the initial surgery.

Sindou [28] in 2007 did not support redo MVD for recurrent $\mathrm{TN}$, describing several attempts but it was felt too hazardous due to the presence of adhesions. Percutaneous treatments were instead recommended. Apfelbaum reported on 289 patients treated with MVD finding recurrent pain in 76 (29.6\%) [29]. Some cases underwent initial exploration in the hope of finding an additional cause but a correctable cause was only found in one in 10 patients and Glycerol chemo neurolysis was recommended rather than repeat posterior fossa exploration [29]. Patients with a prior MVD have been reported to have a higher complication rate if repeat surgery is performed [30]. Trigeminal nerve complications have been reported as high as $32 \%$ while hearing loss is as high as $6.7 \%$ [4] The use of auditory evoked potentials and the routine use of stereotactic navigation has significantly reduced retraction related complications as it allows a more precise trajectory to the cerebello-pontine cistern. We found the surgical access relatively straightforward in the 3 cases as after reopening of the bone flap, the dissection over the cerebellum was not hampered by the petrosal vein as this had been coagulated during the initial surgery. The dissection of the trigeminal nerve due to the adhesions however required meticulous surgical technique.

In our Patient 2, the recurrent TN appears to be directly related to compression of the Silastic sheeting placed at initial surgery into the pre-pontine cistern. While there was initial relief of trigeminal neuralgia, recurrent symptoms occurred. At the second surgery, no recurrent vascular compression was seen but the Silastic sheeting was indenting the nerve (Figure 5b). The patient reported alleviation of trigeminal pain following removal of implant. In both case 1 and case 2, no recurrent arterial compression was seen.

Patient 3 in our report is of recurrent TN 6yrs following MVD in a 69-year-old male. The original surgery was performed in 2006. A temporary recurrent pain occurred after an intraspinal anesthetic in 2012. The pain recurred again in April 2015 when he had a second intraspinal anesthetic. Pain did not improve, and repeat MVD was performed. Recurrent neurovascular compression was found. The development of intractable symptoms following the second intra-spinal anesthetic makes it probable the lumbar puncture associated with the spinal anaesthetic has contributed to the descent of the blood vessels in the pre-pontine and cerebellar cistern. The association between lumbar puncture or intraspinal anesthesia and trigeminal neuralgia has not been previously reported.

\section{Conclusion}

Recurrence of TN symptoms after previous MVD poses a significant management challenge. The development of adhesions between the foreign material and the neighboring vessel and nerve makes the second MVD more difficult. This report describes 3 new cases of recurrent $\mathrm{TN}$ after a substantial period of alleviation of pain after their initial surgery. All 3 cases were treated with repeat MVD after conservative treatment had failed. In each case the etiology of the recurrent compression was different. In Patients 1 and 2 the inserted material, PTFE and silastic caused recurrent compression without any recurrent vascular compression either arterial or venous. In Patient 3, TN was due to recurrent vascular compression, almost certainly due the effects of the spinal anesthetic contributing to the descent of the arterial vessel. These cases support the recommendation that repeat MVD can be highly effective in alleviating recurrent $\mathrm{TN}$ whether the recurrent compression is due to the prosthesis or recurrent vascular compression.

\section{References}

1. Phan K, Rao P, Dexter M (2016) Microvascular decompression for elderly patients with trigeminal neuralgia. J Clin Neuroscience 29: 7-14.

2. Maarbjerg S, Stefano GD, Bendtsen L, Cruccu G (2017) Trigeminal neuralgia-diagnosis and treatment. Cephalalgia 37(7): 648-657.

3. Sade B, Lee JH (2014) Microvascular Decompression for trigeminal neuralgia. Neurosurg Clin N Am 25(4): 743-749.

4. Bick SKB, Eskandar EN (2017) Surgical treatment of trigeminal neuralgia. Neurosurg Clin N Am 28(3): 429-438.

5. Sarsam Z, Garcia-Finana M, Nurmikko TJ, VArma TR, Eldridge P (2010) The long-term outcome of microvascular decompression for trigeminal neuralgia. Br J Neurosurg 24(1): 18-25.

6. Matsushima T, Yamaguchi T, Inoue T, Matsukado K, Fukui M (2000) Recurrent trigeminal neuralgia after microvascular decompression using an interposing technique. Teflon felt adhesion and the sling retraction technique. Acta Neurochir 142(5): 557-561.

7. Barker FG, Jannetta PJ, Bissonette DJ, Larkins MV, Jho HD (1996) The long-term outcome of microvascular decompression for trigeminal neuralgia. N Engl J Med 334(17): 1077-1083. 
8. Burchiel KJ, Clarke H, Haglund M (1988) Long-term efficacy of microvascular decompression in trigeminal neuralgia. J Neurosurgery 69(1): 35-38

9. Tronnier VM, Rasche D, Hamer J, Kienle A-L, Kunze S (2001) Treatment of idiopathic trigeminal neuralgia: Comparison of long-term outcome after radiofrequency rhizotomy and microvascular decompression. Neurosurgery 48(6): 1261-1267.

10. Kureshi SA, Wilkins RH (1988) Posterior fossa reexploration for persistent or recurrent trigeminal neuralgia of hemifacial spasm: Surgical findings and therapeutic implications. Neurosurgery 43(5): 1111-1117.

11. Jannetta PJ, Bissonette DJ (1985) Managment of the failed patient with trigeminal neuralgia. Clin Neurosurgery 32: 334-347.

12. Ugwuanyi U, Kitchen ND (2010) The operative findings in re-do microvascular decompression for recurrent trigeminal neuralgia. $\mathrm{Br}$ Neurosurg 24(1): 26-30.

13. Yang DB, Jian DY, Chen HC, Wang ZM (2015) Second microvascular decompression for trigeminal neuralgia in recurrent cases after microvascular decompression. J Craniofac Surg 26(2): 491-494.

14. Fujimaki T, Hoya K, Sasaki T, Kirino T (1996) Recurrent trigeminal neuralgia caused by an inserted prostehsis: Report of two cases. Acta Neurochir 138(11): 1307-1309.

15. Premsagar IC, Moss T, Coakham HB (1997) Teflon-induced granuloma following treatment of trigeminal neuralgia by microvascular decompression. J Neurosurgery 87(3): 454-457.

16. Lee SH, Levy EI, SCarrow AM, Kassam A, Jannetta PJ (2000) Recurrent trigeminal neuralgia attributable to veins after microvascular decompression. Neurosurgery 46(2): 356-361.

17. Theodros D, Goodwin R, Bender MT, Zhou X, Garzon-Muvdi T, et al. (2017) Efficacy of primary microvascular decompression versus subsequent microvascular decompression for trigeminal neuralgia. J Neurosurgery 126(5): 1691-1697.

18. Nurmikko T, O’Neill F (1997) Evidence-based approach to the treatment of facial pain. Youmans and winn neurological surgery $\left(7^{\text {th }}\right.$ edn $)$, Elsevier USA, pp. 4.

19. Miller JP, Burchiel KJ (2017) Microvascular decompression for trigeminal neuralgia. Youmans and winn neurological surgery $\left(7^{\text {th }}\right.$ edn), Elsevier, USA, pp. 1420-1426.
20. Bederson JB, Wilson CB (1989) Evaluation of microvascular decompression and partial sensory rhizotomy in 252 cases of trigeminal neuralgia. J Neurosurgery 71(3): 359-367.

21. Rath SA, Klein HJ, Richter HP (1996) Findings and long-term results of subsequent operations after failed microvascular decompression for trigeminal neuralgia. Neurosurgery 39(5): 933-938.

22. Choo DY, Chang CG, Wang YC, Wang FH, Shen CC, et al. (1994) Repeat operations in failed microvascular decompression for trigeminal neuralgia. Neurosurgery 353(4): 665-669.

23. Miller JP, Magill ST, Acar F, Burchiel KJ (2009) Predictors of long-term success after microvascular decompression for trigeminal neuralgia. Neurosurgery 110(4): 620-626.

24. Illingworth NM (1995) Trigeminal neuralgia treated by microvascular decompression: a long-term follow-up study. Br J Neurosurg 9(1): 13-20.

25. Yamaki T, Hashi K, Niwa J, TAnabe S, Nakagawa T, et al. (1992) Results of reoperation for failed microvascular decompression. Acta Neurochir (Wien) 115(1-2): 1-7.

26. Ammar A, Lagenaur C, Jannetta P (1990) Neural tissue compatibility of teflon as an implant material for microvascular decompression. Neurosurg Rev 13(4): 299-303.

27. Cho DY, Chang CG, Wang YC, Wang FH, Shen CC (1994) Repeat operations in failed microvascular decompression for trigeminal neuralgia. Neurosurgery 35(4): 6665-669

28. Sindou M, Leston J, Decullier E, Chapuis F (2007) Microvascular decompression for primary trigeminal neuralgia: long-ter effectiveness and prognostic factors in a series of 362 consecutive patients with clear-cut neurovascular conflicts who underwent pure decompression. J Neurosurgery 107(6): 1144-1153.

29. Apfelbaum RI (1983) Surgery for tic douloreux. Clin Neurosurgery 31: 351-368.

30. Chakravarthi S, Ghanta R, Kattimani V (2011) Microvascular decompression treatment for trigeminal neuralgia. Journal of Craniofacial Surgery 22(3): 894-898.

For possible submissions Click below: 